CONFORMAL GEOMETRY AND DYNAMICS

An Electronic Journal of the American Mathematical Society

Volume 10, Pages 285-287 (October 5, 2006)

S $1088-4173(06) 00154-8$

\title{
FORMAL ADJOINTS AND A CANONICAL FORM FOR LINEAR OPERATORS
}

\author{
MICHAEL G. EASTWOOD AND A. ROD GOVER
}

\begin{abstract}
We describe a canonical form for linear differential operators that are formally self-adjoint or formally skew-adjoint.
\end{abstract}

Suppose $E$ and $F$ are smooth vector bundles on an oriented smooth manifold $M$. Let $v o l$ denote the bundle of volume forms on $M$. The formal adjoint of a linear differential operator $L: E \rightarrow F$ is the differential operator $L^{*}: F^{*} \otimes v o l \rightarrow E^{*} \otimes v o l$ characterised by the equation

$$
\int_{M}\left\langle L^{*} \sigma, \tau\right\rangle=\int_{M}\langle\sigma, L \tau\rangle \quad \text { for } \sigma \in \Gamma\left(M, F^{*} \otimes v o l\right) \text { and } \tau \in \Gamma_{*}(M, E) .
$$

Here all sections are assumed sufficiently smooth and $\Gamma_{*}$ indicates the space of compactly supported sections. If $F=E^{*} \otimes \mathrm{vol}$, then $L^{*}: E \rightarrow F$ and there is a canonical decomposition

$$
L=L_{+}+L_{-}=\frac{1}{2}\left[L+L^{*}\right]+\frac{1}{2}\left[L-L^{*}\right]
$$

into self-adjoint and skew-adjoint parts. Henceforth let us assume that $M$ is equipped with a preferred volume form and a compatible torsion-free connection $\nabla$ (e.g. $M$ is Riemannian).

Suppose that $E$ is trivial. Then the preferred volume form trivialises $F$, and so $L$ may be viewed as taking functions to functions and written in terms of the given connection. A formula for its adjoint is determined by integration by parts. Suppose, for example, that $L$ is second order. Then we may write

$$
L=S^{a b} \nabla_{a} \nabla_{b}+T^{b} \nabla_{b}+R,
$$

where the tensor $S^{a b}$ is symmetric. Adopting the convention that $\nabla_{a}$ acts on everything to its right, we can re-express $L$ in the form

$$
L=\nabla_{a} S^{a b} \nabla_{b}+\left(\tilde{T}^{b} \nabla_{b}+\nabla_{b} \tilde{T}^{b}\right)+\tilde{R}
$$

where the tensor $\tilde{T}$ and scalar field $\tilde{R}$ are given by $\tilde{T}^{b}=\frac{1}{2}\left(T^{b}-\left(\nabla_{a} S^{a b}\right)\right)$ and $\tilde{R}=R-\left(\nabla_{b} \tilde{T}^{b}\right)$. This is congenial since clearly

$$
L^{*}=\nabla_{a} S^{a b} \nabla_{b}-\left(\tilde{T}^{b} \nabla_{b}+\nabla_{b} \tilde{T}^{b}\right)+\tilde{R} .
$$

Received by the editors July 18, 2006.

2000 Mathematics Subject Classification. Primary 58J70; Secondary 53A30.

Key words and phrases. Adjoints, differential operators, conformal invariance.

The first author is supported by the Australian Research Council.

The second author expresses appreciation for support by the New Zealand Institute for Mathematics and its Applications and the Royal Society of New Zealand (Marsden Grant 02-UOA-108).

(C)2006 American Mathematical Society Reverts to public domain 28 years from publication 
In particular,

$$
L_{+}=\nabla_{a} S^{a b} \nabla_{b}+\tilde{R} \quad \text { and } \quad L_{-}=\tilde{T}^{b} \nabla_{b}+\nabla_{b} \tilde{T}^{b} .
$$

By an obvious inductive argument this generalises immediately to give the following result.

Theorem 1. A self-adjoint $k$ th order linear differential operator taking functions to functions on $M$ has even order and may be canonically written in the form:

$$
\sum_{i=0}^{k / 2} \underbrace{\nabla_{a} \cdots \nabla_{b}}_{i} S_{(i)}^{a \cdots b c \cdots d} \underbrace{\nabla_{c} \cdots \nabla_{d}}_{i}
$$

for suitable symmetric tensors $S_{(i)}^{a \cdots d}$. A skew-adjoint $k$ th order linear differential operator taking functions to functions on $M$ has odd order and may be canonically written in the form:

$$
\sum_{i=0}^{(k-1) / 2} \underbrace{\nabla_{a} \cdots \nabla_{b}}_{i}\left(\nabla_{c} A_{(i)}^{a \cdots b c d \cdots e}+A_{(i)}^{a \cdots b c d \cdots e} \nabla_{c}\right) \underbrace{\nabla_{d} \cdots \nabla_{e}}_{i},
$$

for suitable symmetric tensors $A_{(i)}^{a \cdots e}$.

Suppose now that $E$ is a vector bundle (possibly a tensor bundle) with connection on $M$ and write $\nabla$ to indicate the coupled tensor-vector bundle connection. Let us use upper case Greek indices as abstract indices for the bundle $E$ and its dual. Then, for example, operators $L: E \rightarrow E^{*}$ may be written $L_{\Psi \Phi}: E^{\Phi} \rightarrow E_{\Psi}$. Since the tensor product $E^{*} \otimes E^{*}$ decomposes canonically into symmetric and skewsymmetric parts, it follows easily that the above generalises as follows. We write $[\ell]$ to indicate the integer part of a real number $\ell$.

Theorem 2. A self-adjoint (respectively skew-adjoint) $k$ th order linear differential operator $L_{\Psi \Phi}: E^{\Phi} \rightarrow E_{\Psi}$ on $M$ may be canonically written in the form:

$$
\begin{aligned}
\sum_{i=0}^{[k / 2]} & \underbrace{\nabla_{a} \cdots \nabla_{b}}_{i} S_{\Psi \Phi(i)}^{a \cdots b c \cdots d} \underbrace{\nabla_{c} \cdots \nabla_{d}}_{i} \\
& +\sum_{i=0}^{[(k-1) / 2]} \underbrace{\nabla_{a} \cdots \nabla_{b}}_{i}\left(\nabla_{c} A_{\Psi \Phi(i)}^{a \cdots b c d \cdots e}+A_{\Psi \Phi(i)}^{a \cdots b c d \cdots e} \nabla_{c}\right) \underbrace{\nabla_{d} \cdots \nabla_{e}}_{i},
\end{aligned}
$$

where the sections $S_{\Psi \Phi(i)}^{a \cdots d}$ are symmetric over the tensor indices and symmetric (respectively skew-symmetric) over the pair $\Psi \Phi$ of vector bundle indices; the sections $A_{\Psi \Phi(i)}^{a \cdots e}$ are symmetric over the tensor indices and skew-symmetric (respectively symmetric) over the pair $\Psi \Phi$ of vector bundle indices.

Here is an example application. Suppose that $P$ is a self-adjoint linear differential operator $P$ of order $k>0$ that takes functions to functions on $M$. Write $\tilde{P}$ for the modified operator obtained by subtracting from $P$ the scalar part obtained by applying $P$ to $f \equiv 1$. Then $\tilde{P}$ is formally self-adjoint and takes the form $f \mapsto \nabla_{a}\left(Q^{a b}\left(\nabla_{b} f\right)\right)$ for a suitable differential operator $Q: T^{*} M \rightarrow T M$. Since the construction of $\tilde{P}$ is canonical, $\tilde{P}$ enjoys the same naturality and/or invariance properties as does $P$.

A good example arises in conformal geometry. Suppose $M$ is an oriented conformal manifold of even dimension $2 m$. Let $L$ denote any conformally invariant 
operator on functions that, with respect to any Riemannian metric in the conformal class, takes the form

$$
L=\Delta^{m}+\text { lower order terms. }
$$

Since $L$ takes functions to volume forms, so does its formal adjoint. The self-adjoint part $L_{+}$of $L$ is therefore also conformally invariant. As a conformal analogue of $\Delta^{m}$ (in the sense of [3]), we may as well replace $L$ by $L_{+}$. Then by Theorem 1 this, in turn, may be replaced by a self-adjoint conformally invariant modification of the form $f \mapsto \nabla_{a}\left(Q^{a b}\left(\nabla_{b} f\right)\right)$ for a suitable $(n-2)$ nd order differential operator $Q: \Lambda^{1} \rightarrow \Lambda^{n-1}$. Thus, we obtain the following result conjectured to us by Tom Branson.

Theorem 3. Any conformally invariant linear differential operator of the form (11) admits a self-adjoint conformally invariant modification of the form $f \mapsto$ $\nabla_{a}\left(Q^{a b}\left(\nabla_{b} f\right)\right)$ for a suitable $(n-2) n d$ linear order differential operator $Q: \Lambda^{1} \rightarrow$ $\Lambda^{n-1}$

The motivation for Branson's conjecture came from the case of the sphere where the form of the operator may be verified directly. On the sphere, the operator controls the embedding $L_{m}^{2} \hookrightarrow e^{L}$ (Orlitz class) as a limiting case of the sharp Sobolev embeddings $L_{r}^{2} \hookrightarrow L^{\frac{2 m}{m-r}}$ for $r<n / 2$ (equivalently, comparing an $L^{q}$ norm with the complementary series norm); see [1] for further discussion. In [4, Graham, Jenne, Mason, and Sparling established the existence of operators of the form (11). More recently it has been shown, using scattering theory [5] (and alternatively via the Fefferman-Graham ambient metric [2]) that the particular operators constructed in 4. are, in fact, already formally self-adjoint and have the form given in Theorem 3 ,

\section{REFERENCES}

[1] T.P. Branson, Sharp inequalities, the functional determinant, and the complementary series, Trans. A.M.S. 347 (1995), 3671-3742. MR.1316845(96e:58162)

[2] T. Branson and A.R. Gover, Conformally invariant operators, differential forms, cohomology and a generalisation of Q-curvature, Comm. Partial Differential Equations 30 (2005), 16111669. MR2182307

[3] M.G. Eastwood and J.W. Rice, Conformally invariant differential operators on Minkowski space and their curved analogues, Commun. Math. Phys. 109 (1987), 207-228. MR0880414 (89d:22012)

[4] C.R. Graham, R. Jenne, L.J. Mason, and G.A.J. Sparling, Conformally invariant powers of the Laplacian, I: Existence, Jour. Lond. Math. Soc. 46 (1992), 557-565. MR1190438 (94c:58226)

[5] C.R. Graham and M. Zworski, Scattering matrix in conformal geometry, Invent. Math. 152 (2003), 89-118. MR1965361 (2004c:58064)

Department of Pure Mathematics, University of Adelaide, South Australia 5005

E-mail address: meastwoo@maths.adelaide.edu.au

Department of Mathematics, The University of Auckland, Private Bag 92019, AuckLAND 1, NEW ZEALAND

E-mail address: gover@math.auckland.ac.nz 